\title{
5 Research Square

\section{Socio-demographic characteristics influencing knowledge, attitude and preventive practices of COVID-19 among Ghanaians: A cross-sectional study}

Philip Apraku Tawiah ( $\square$ ptawiah@uhas.edu.gh )

University of Health and Allied Sciences https://orcid.org/0000-0003-0212-195X

Kingsley Arhin-Wiredu

Sunyani Municipal Health Directorate

Kwabena Oppong

Kumasi Academy

Bernard Nii Torgbor

Valco Hospital

Phenehance Effah Konadu

Dunkwa-On-Offin Health Directorate

Albert Abaka-Yawson

University of Health and Allied Sciences

\section{Research Article}

Keywords: Novel coronavirus, COVID-19, Knowledge, Attitudes, Practices, Ghana

Posted Date: October 6th, 2021

DOI: https://doi.org/10.21203/rs.3.rs-859719/v1

License: (1) This work is licensed under a Creative Commons Attribution 4.0 International License. Read Full License 


\section{Abstract \\ Background}

In recent times, the novel coronavirus disease, obviously, is one of the most challenging public health burden. Low-and-middle-income countries including Ghana have not been spared by the infection. Several measures are being undertaken by countries to control and prevent the future waves of the disease. Previous studies have established the importance of knowledge, attitude and practices towards an infection as remedy for controlling the spread of such infections. However, further studies are required to ascertain the sociodemographic characteristics influencing the knowledge, attitude and preventive measures of COVID-19.

\section{Methods}

This study investigated the socio-demographic characteristics influencing knowledge, attitude and practices towards COVID-19 infection among the Ghanaian population. A cross-sectional design was adopted to recruit 369 participants for the study by means of convenience and snowball sampling methods. With the use of an electronic semi-structured questionnaire and social media platforms, an online survey was conducted from March 26 - May 1, 2020. Descriptive statistics, independent t-test, chi-square test, one-way analysis of variance (ANOVA) and linear regression were used to analyze data.

\section{Results}

The average mean percentage of good knowledge, positive attitudes and good preventive practices regarding the novel coronavirus disease was $83.2 \%, 88.2 \%$ and $69.2 \%$, correspondingly. In relation to occupation, there was significant difference $(t=-2.984, p$-value $=0.0030)$ in COVID-19 knowledge. Income level was significantly associated with attitudes towards COVID-19. Male gender (vs female, $\beta=-1.276$, $p$-value $=<0.00001$ ), less than $\$ 500$ income earners (vs above $\$ 1000, \beta=-0.845$, $p$-value $=0.017$ ) were associated with lower COVID-19 preventive practice score while being the $42-64$ age category (vs 18-25-year group, $\beta=1.128$, $p$-value $=$ 0.028 ), health worker (vs non-health worker, $\beta=0.816$, $p$-value $=0.001$ ) and belonging to Ga ethnic group (vs Akan ethnic group, $\beta=0.89$, $p$-value $=0.027$ ) were associated with higher scores.

\section{Conclusion}

Although this study found higher knowledge and attitudes toward the novel coronavirus disease, it did not reflect in higher preventive practices towards the infection. In our fight against this infection in Ghana, COVID19 preventive measure educational campaigns targeting male gender and low-income earners should be considered and implemented.

\section{Introduction}

Since the emergence of the novel coronavirus disease (COVID-19) in December 2019 from Wuhan city in China, it has spread to many other countries around the World including those on the African continent (1). 
The World Health Organization (WHO) on January 30, 2020 declared the outbreak of the disease as Public Health Emergency of International Concern, serving as a clarion call to the international community to provide political, financial and technical support to a public health emergency (2). Subsequently, COVID-19 was declared as a pandemic on March 11, 2020 due to its widespread across the globe (3).

In Ghana, the Ministry of Health (MOH) in a release on March 12, 2020 announced the first two confirmed cases of COVID-19 (4). After the pronouncement of these initial cases, the Government of Ghana (GoG) put in place numerous measures including closing of borders, schools, churches, mosques and ban of all social gatherings (5). Citizens were also advised by GoG to practice precautionary measures such as washing of hands with soap, sanitizing, social distancing among others. Notwithstanding, as of May 1, 2020, Ghana was among the top African countries who had recorded most cases of COVID-19 with 2074 confirmed cases, 212 recoveries and 17 deaths $(6)$.

The battle against COVID-19 by GoG and Ghanaians as a whole continued through various phases with strict measures such as partial lockdown in various parts of the country such as Greater Accra, Greater Kumasi and Kasoa Metropolis for three weeks. This was accompanied by compulsory wearing of nose masks, particularly in the Greater Accra region. In the wake of all these measures put in place by GoG, people's adherence to these measures are significant and is chiefly affected by Knowledge, Attitude and Practices (KAP) towards COVID19. This is in accordance to the KAP theory $(7,8)$.

According to some studies $(9,10)$ conducted during the SARS outbreak in 2003 , knowledge and attitudes toward infectious diseases like COVID-19 has a causal connection with level of panic emotion among the general population and can consequently complicate the efforts made to prevent the transmission of the disease. Therefore, it is imperative to have some basic knowledge about the COVID-19 disease and its modes of transmission, as well as basic hygiene principles, in order to reduce the panics related to the disease and its spread.

Currently, studies have been done in some countries such as China (11), Egypt (12), Iran (13), Kenya (14) on KAP towards COVID-19 among the general population. However, the situation in Ghana has not been adequately assessed, especially the investigation of socio-demographic characteristics influencing the knowledge, attitude and practices of the COVID-19 pandemic. In view of this and all the precautionary measures put in place by the GoG, including daily education on television, radio, print and social media platforms concerning COVID-19, the study investigated knowledge, Attitude and Practice towards the novel coronavirus disease among Ghanaians. It also ascertain the socio-demographic characteristics influencing the knowledge, attitude and preventive practices of the novel coronavirus disease in the country.

\section{Methods}

\section{Study design}

A cross sectional study was conducted from 26 March to 1st May, 2020. The study adopted an online survey approach among Ghanaians, due to the restricted movement imposed on Ghanaians during the COVID 19 
pandemic period. Social network platforms such as WhatsApp, Facebook, Instagram and twitter were used as the channels for reaching people to respond to the survey.

\section{Study area}

The study was conducted among Ghanaians through online social media platforms. Ghana is a West African Country bordered by the countries; Burkina Faso, Ivory Coast and Togo in the north, west and east respectively. However, the south is bordered by the Atlantic Ocean. Ghana is divided into 16 administrative regions with Accra as the national city. The population is estimated to reach 31,072,940 in the year 2020 with Akans and Christians forming $47.5 \%$ and $71.2 \%$ of the population correspondingly. The health system is manned by the Ghana Health Service offering primary, secondary and tertiary health care at the various regions.

\section{Sample size and sampling procedure}

The Centre for Disease Control Epi Info Software 7.2.3.1 was used to compute the minimum sample size required for the study. An expected frequency of $50 \%$ was used mainly because there was limited study quantifying the proportion of the population with knowledge on COVID-19. With alpha value of $5 \%$ at a confidence level 95\%, a total sample size of 384 was computed. The study assumed a non-response rate of $5 \%$, which resulted in a total sample size of 403 . However, a total of 369 respondents took part in the study, with a response rate of $91.6 \%$. Due to the national lockdown, respondents for the study were recruited through convenience and snowball sampling methods.

\section{Inclusion criteria}

- Ability to read and operate a smart phone

- Access to the internet and social media

- Eighteen (18) years and above

- Willingness to participate in the study

\section{Exclusion criteria}

- Below 18 years

- Unwillingness to participate in the study

\section{Data collection technique}

An online survey tool was designed with google forms comprising four sections. These sections included demographic characteristics, knowledge on COVID-19, attitude and practices towards COVID 19 among the Ghanaian population. The tool was developed in English language. The questionnaires were multiple choice and limited format. The data collection instrument was reviewed by professionals including medical officers, medical laboratory scientists, disease control officers and disease control officers to ascertain its reliability and validity. This was followed by a pretesting. The tool was shared with authors' social contacts on WhatsApp, Facebook, twitter and Instagram. Respondents were also encouraged to share with their networks after completing the survey.

\section{Data Processing and Analysis}


Collected data from google sheet was exported to excel for cleaning and imported into Stata/IC 15 statistics/data analysis software for data analysis. Categorical variables were presented as frequencies and percentages. Analysis of the KAP was done using scaler scoring method. Questions with multiple responses were assigned 1 point and 0 for unanswered questions. Other questions with three responses were assigned 0,1 and 2. For each respondent, a maximum score of 22, 6 and 13 were awarded for correctly answering all questions, good attitudes and good practices in that order. Independent t test, one-way ANOVA and chi square test were used to analyze and compare demographic characteristics to knowledge, practices and attitude to know statistically significant variables at $p$ values of $5 \%$. Linear regression model was used to analyze factors associated with knowledge, attitude and practices on COVID-19.

\section{Results}

\subsection{Demographic characteristics of respondents}

The study received participation from three hundred and thirty-nine (369) people. Out of this, majority, 231 (62.6\%) were males. A greater number, 156 (42.3\%) of participants were within the 26-33 age bracket while the least, $26(7.1 \%)$ were in the 42-64 age category. More than half, 207 (56.1\%) were not health workers. Almost all, 356 (96.5) were Christians and had attained tertiary education. Also, most, 221 (60.0\%) and 215 (58.3) of study respondents earned an average income of more than $\$ 1000$ and were Akans, respectively. Again, majority, 123 (33.3\%) respondents were residents of the Greater Accra region, this was followed by Ashanti and Eastern region. (Table 1) 
Table 1

Demographic Characteristics of Respondents by occupational status

\begin{tabular}{|c|c|c|c|}
\hline \multirow[t]{2}{*}{ Characteristics } & Non-health worker & Health worker & Total \\
\hline & 207 (56.10\%) & $162(43.90 \%)$ & $369(100.00 \%)$ \\
\hline \multicolumn{4}{|l|}{ Gender } \\
\hline Female & 75 (36.23) & 63 (38.89) & $138(37.40)$ \\
\hline Male & 132 (63.77) & 99 (61.11) & $231(62.60)$ \\
\hline \multicolumn{4}{|l|}{ Age } \\
\hline $18-25$ & $56(27.05)$ & 36 (22.22) & $92(24.93)$ \\
\hline $26-33$ & $84(40.58)$ & $72(44.44)$ & $156(42.28)$ \\
\hline $34-41$ & 56 (27.05) & 39 (24.07) & 95 (25.75) \\
\hline $42-64$ & $11(5.31)$ & $15(9.26)$ & $26(7.05)$ \\
\hline \multicolumn{4}{|c|}{ Educational level } \\
\hline Secondary & $13(6.28)$ & $0(0.00)$ & $13(3.52)$ \\
\hline Tertiary & 194 (93.72) & $162(100.00)$ & $356(96.48)$ \\
\hline \multicolumn{4}{|l|}{ Religion } \\
\hline Christianity & $202(97.58)$ & 154 (95.06) & $356(96.48)$ \\
\hline Islamic & $4(1.93)$ & $6(3.70)$ & $10(2.71)$ \\
\hline Others & $1(0.48)$ & $2(1.23)$ & $3(0.81)$ \\
\hline \multicolumn{4}{|l|}{ Income level ( $(\mathbf{)})$} \\
\hline$>1000$ & $116(56.04)$ & $105(64.81)$ & $221(59.89)$ \\
\hline $500-1000$ & 41 (19.81) & 32 (19.75) & 73 (19.78) \\
\hline$<500$ & $50(24.15)$ & $25(15.43)$ & 75 (20.33) \\
\hline \multicolumn{4}{|l|}{ Ethnicity } \\
\hline Akan & $126(60.87)$ & 89 (54.94) & 215 (58.27) \\
\hline Ewe & 33 (15.94) & $29(17.90)$ & $62(16.80)$ \\
\hline $\mathrm{Ga}$ & $20(9.66)$ & $13(8.02)$ & 33 (8.94) \\
\hline Others & 28 (13.53) & 31 (19.14) & 59 (15.99) \\
\hline Region of reside & & & \\
\hline
\end{tabular}

Others* refers to Ahafo, Bono East, North East, Northern, Oti, Savannah, Upper East, Upper West and Western North regions. 


\begin{tabular}{|llll|}
\hline Characteristics & Non-health worker & Health worker & Total \\
\cline { 2 - 4 } & $\mathbf{2 0 7}(\mathbf{5 6 . 1 0})$ & $\mathbf{1 6 2 ( 4 3 . 9 0 \% )}$ & $\mathbf{3 6 9}(\mathbf{1 0 0 . 0 0 \% )}$ \\
\hline Ashanti & $21(\mathbf{1 0 . 1 4 )}$ & $31(19.14)$ & $52(14.09)$ \\
\hline Bono & $6(2.90)$ & $17(10.49)$ & $23(6.23)$ \\
\hline Central & $16(7.73)$ & $12(7.41)$ & $28(7.59)$ \\
\hline Eastern & $36(17.39)$ & $9(5.56)$ & $45(12.20)$ \\
\hline Greater Accra & $85(41.06)$ & $38(23.46)$ & $123(33.33)$ \\
\hline Volta & $14(6.76)$ & $26(16.05)$ & $40(10.84)$ \\
\hline Western & $11(5.31)$ & $4(2.47)$ & $15(4.07)$ \\
\hline $\begin{array}{l}\text { Others* } \\
\text { Others } \\
\text { Western Ners to Ahafo, Bono East, North East, Northern, Oti, Savannah, Upper East, Upper West and }\end{array}$ \\
\hline
\end{tabular}

\subsection{Participants Knowledge on COVID-19}

The average mean of knowledge on COVID-19 among study participants was $18.3 \pm 2.36$ out of a maximum score of 22, showing a mean percentage good knowledge of $83.2 \%$ (Fig. 1). The correct answer rate of questions (K1-K12) relating to knowledge on COVID-19 ranged from 4.3-99.2\%. However, correct answer rate to questions (K1-K7) that required a single answer ranged from 89.4-99.2\%. With regards to questions (K8$\mathrm{K} 12)$ that required more than one correct answer, the rate of identifying all correct answers ranged from 34.791.9\%. (Table 2) 
Table 2

Participant's knowledge on COVID-19

Questions/Statements

$N(\%)$

K1. COVID-19 spreads by breathing droplets containing the virus from an infected person

No

Yes*

K2. A person who tests positive for COVID-19 but shows no symptoms cannot spread the virus

True

$39(10.57)$

False*

K3. It is not important for children and youth to measures to prevent COVID-19

True

False*

K4. It is recommended that in order to stay safe, you must wash your hands frequently

True*

False

K5. There is a specific approved antiviral treatment for COVID-19?

Yes

No*

K6. Are there approved vaccines currently on the market to prevent COVID-19?

Yes

No*

K7. Persons with COVID-19 cannot infect others with the virus if a fever is not present

True

False*

K8. Which people are at risk of getting infected with COVID-19?

Don't know

$2(0.54)$

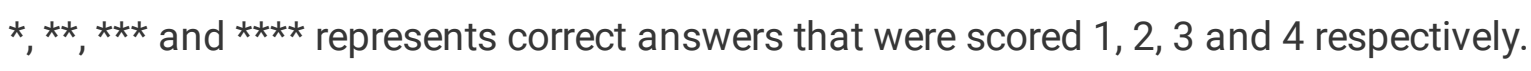




\section{Questions/Statements}

The aged and people with chronic disease*

Everyone $e^{\star \star}$

K9. Common clinical symptoms of COVID-19 are

Don't know

Fever*

Fever and fatigue ${ }^{\star \star}$

Fever, fatigue and dry cough ${ }^{\star \star \star}$

Fever, fatigue, dry cough and sore throat ${ }^{\star \star \star *}$

\section{K 10. Critical cases of COVID-19 are common among}

Don't know

Elderly*

Elderly and people who are obese.**

Elderly, people who are obese and patients with chronic illness ${ }^{\star \star \star}$

\section{$\mathrm{K} 11$. Clinical symptoms associated with severe cases of COVID-19 includes}

Don't know

Difficulty in breathing*

Difficulty in breathing and high fever**

Difficulty in breathing, high fever and pneumonia***

\section{K 12. In your opinion COVID-19 can be prevented by}

Don't know

Avoiding crowded places*

Avoiding crowded places and not greeting through handshaking**

Avoiding crowded places, not greeting through handshaking and not drinking alcoholic beverages ${ }^{\star \star *}$

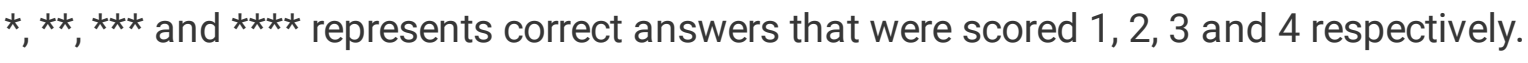

\subsection{Attitudes of participant's towards COVID-19}


The average mean of participant's attitude towards COVID-19 was $5.29 \pm 0.88$ out of a maximum score of 6 , representing a mean percentage positive attitude of $88.2 \%$. (Fig. 1) Majority, $61.5 \%$ of above $c 1000$ per month income earners had good attitudes towards the novel disease. (Fig. 2) Based on the questions to ascertain attitudes towards COVID-19, good attitude rate ranged from 75.1-99.7\%. Majority of participants (77.0\%) thought it was safe to get closer to a person who has previously suffered from COVID-19. Almost all (99.7\%) participants claimed it was significant to observe the health guidelines. Majority, $91.3 \%$ and $90.2 \%$ of participants did not believe COVID-19 is a spiritual disease and deception, respectively. Many, $75.1 \%$ and $95.4 \%$ of the respondents also were confident the Government of Ghana could win the battle against COVID19 , and successfully control the pandemic correspondingly. (Table 3)

Table 3

Attitudes of participant's towards COVID-19

\section{Questions on attitudes toward COVID-19 (good attitudes rate, percentage of total Options sample \%)}

A1. Do you agree that COVID-19 could be successfully controlled? (95.4)

Disagree, Agree*, don't know

A2. Do you have confidence that the Government of Ghana can win the battle against COVID-19? (75.1)

No, Yes*, don't know

A3. Do you think it is safe to get close to a person who suffered previously from COVID-19? (77.0)

No, Yes*, don't know

A4. Do you think it is important to observe the health guidelines (including social distancing and wearing of mask)? (99.7)

No, Yes*, don't know

A5. Is COVID-19 a spiritual disease? (91.3)

No*, Yes, don't know

A6. Is COVID-19 a hoax (deception)? (90.2) No*, Yes, don't know

* corresponds to options perceived as good attitude towards COVID-19.

\subsection{Preventive practices against COVID-19 by study participants}

The average mean of COVID-19 preventive practices was $9.0 \pm 2.18$ out of a maximum score of 13 , making up a mean percentage good preventive practice of $69.2 \%$. (Fig. 1) Also, $60.1 \%$ of males were involved in good practices towards the novel disease. (Fig. 3) Most participants, 179 (48.5\%) and 299 (81.0\%) always wore masks and avoided crowded places, respectively. Majority of participants, 359 (97.3\%) washed their hands with soap under running water; nonetheless, only one-third, $123(33.3 \%)$ of them did the washing of hands more than 10 times in a day. Also, a little above one-third of the participants, 130 (35.2\%) disinfected their hands with alcohol-based sanitizers more than 10 times daily. Again, many of the participants admitted they sometimes touched their face, nose and mouth. (Table 4) 
COVID-19 practices by study participants

\begin{tabular}{|c|c|}
\hline Questions/Statements & $N(\%)$ \\
\hline \multicolumn{2}{|c|}{ P1. Do you wear masks when leaving home? } \\
\hline Never & $35(9.49)$ \\
\hline Sometimes* & $155(42.01)$ \\
\hline Always** & $179(48.51)$ \\
\hline \multicolumn{2}{|c|}{ P2. Do you wash your hands with soaps under running water for at least 20 seconds? } \\
\hline No & $10(2.71)$ \\
\hline Yes* & $359(97.29)$ \\
\hline \multicolumn{2}{|c|}{ P3. How frequently do you disinfect your hands with alcohol-based sanitizers? } \\
\hline None & $17(4.61)$ \\
\hline $1-4$ times* & $124(33.60)$ \\
\hline $5-10$ times $^{\star \star}$ & $98(26.56)$ \\
\hline 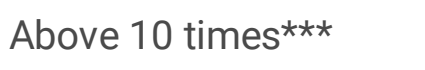 & $130(35.23)$ \\
\hline \multicolumn{2}{|c|}{ P4. How often do you touch your face, nose and mouth? } \\
\hline Not at all & $42(11.38)$ \\
\hline Sometimes * & $262(71.00)$ \\
\hline Most of the times** & $65(17.62)$ \\
\hline \multicolumn{2}{|c|}{ P5. Do you avoid crowded places in recent times (social distancing)? } \\
\hline Never & $1(0.27)$ \\
\hline Sometimes* & $69(18.70)$ \\
\hline Always** & $299(81.03)$ \\
\hline \multicolumn{2}{|c|}{ P6. How many times in a day do you wash your hands? } \\
\hline None & $3(0.81)$ \\
\hline $1-4$ times* & $116(31.44)$ \\
\hline $5-10$ times ${ }^{\star \star}$ & $127(34.42)$ \\
\hline Above 10 times ${ }^{\star \star \star}$ & $123(33.33)$ \\
\hline
\end{tabular}




\subsection{Association Between Practices / Attitudes towards COVID- 19 and Demographic Characteristics}

In relation to demographic characteristics, gender was significantly (Pearson chi2 $=7.34, p$-value $=0.007$ ) associated with practices towards COVID-19, while income level of participants was also significantly (Pearson chi2 $=8.48, \mathrm{p}$-value $=0.007$ ) associated with attitudes towards the COVID-19 pandemic. $($ Table 5$)$ 
Table 5

Association Between Practices, Attitudes towards COVID-19 and Demographic Characteristics

\begin{tabular}{|c|c|c|c|c|c|c|c|c|}
\hline \multirow[t]{2}{*}{ Characteristics } & \multicolumn{4}{|l|}{ Practices } & \multicolumn{4}{|l|}{ Attitudes } \\
\hline & Poor & Good & Chi2 & p-value & Poor & Good & Chi2 & $\begin{array}{l}\mathrm{p}- \\
\text { value }\end{array}$ \\
\hline Gender & & & 7.34 & $0.007 a^{*}$ & & & 0.00 & $0.993^{a}$ \\
\hline Female & $8(5.80)$ & $\begin{array}{l}130 \\
(94.20)\end{array}$ & & & $6(4.35)$ & $\begin{array}{l}132 \\
(95.65)\end{array}$ & & \\
\hline Male & $\begin{array}{l}35 \\
(15.15)\end{array}$ & $\begin{array}{l}196 \\
(84.85)\end{array}$ & & & $\begin{array}{l}10 \\
(4.33)\end{array}$ & $\begin{array}{l}221 \\
(95.67)\end{array}$ & & \\
\hline Age & & & 4.66 & 0.197 & & & 7.87 & 0.066 \\
\hline $18-25$ & $\begin{array}{l}14 \\
(15.22)\end{array}$ & 78 (84.78) & & & $8(8.70)$ & $84(91.30)$ & & \\
\hline $26-33$ & $\begin{array}{l}21 \\
(13.46)\end{array}$ & $\begin{array}{l}135 \\
(86.54)\end{array}$ & & & $7(4.49)$ & $\begin{array}{l}149 \\
(95.51)\end{array}$ & & \\
\hline $34-41$ & $6(6.32)$ & 89 (93.68) & & & $1(1.05)$ & 94 (98.95) & & \\
\hline $42-64$ & $2(7.69)$ & $24(92.31)$ & & & $0(0.00)$ & $\begin{array}{l}26 \\
(100.00)\end{array}$ & & \\
\hline Occupation & & & 1.61 & $0.205_{a}$ & & & 2.43 & 0.132 \\
\hline $\begin{array}{l}\text { Non-health } \\
\text { worker }\end{array}$ & $\begin{array}{l}28 \\
(13.53)\end{array}$ & $\begin{array}{l}179 \\
(86.47)\end{array}$ & & & $\begin{array}{l}12 \\
(5.80)\end{array}$ & $\begin{array}{l}195 \\
(94.20)\end{array}$ & & \\
\hline Health worker & $15(9.26)$ & $\begin{array}{l}147 \\
(90.74)\end{array}$ & & & $4(2.47)$ & $\begin{array}{l}158 \\
(97.53)\end{array}$ & & \\
\hline Educational & & & 0.21 & 0.540 & & & 0.61 & 1.000 \\
\hline Secondary & $1(7.69)$ & $12(92.31)$ & & & $0(0.00)$ & $\begin{array}{l}13 \\
(100.00)\end{array}$ & & \\
\hline Tertiary & $\begin{array}{l}42 \\
(11.80)\end{array}$ & $\begin{array}{l}314 \\
(88.20)\end{array}$ & & & $\begin{array}{l}16 \\
(4.49)\end{array}$ & $\begin{array}{l}340 \\
(95.51)\end{array}$ & & \\
\hline Religion & & & 1.78 & 0.734 & & & 0.61 & 1.000 \\
\hline Christianity & $\begin{array}{l}43 \\
(12.08)\end{array}$ & $\begin{array}{l}313 \\
(87.92)\end{array}$ & & & $\begin{array}{l}16 \\
(4.49)\end{array}$ & $\begin{array}{l}340 \\
(95.51)\end{array}$ & & \\
\hline Islamic & $0(0.00)$ & $\begin{array}{l}10 \\
(100.00)\end{array}$ & & & $0(0.00)$ & $\begin{array}{l}10 \\
(100.00)\end{array}$ & & \\
\hline Others & $0(0.00)$ & $3(100.00)$ & & & $0(0.00)$ & $3(100.00)$ & & \\
\hline Income level ( $(\zeta)$ & & & 2.03 & $0.363_{a}$ & & & 8.48 & $0.007 *$ \\
\hline
\end{tabular}

Others* refers to Ahafo, Bono East, North East, Northern, Oti, Savannah, Upper East, Upper West and Western North regions. 


\begin{tabular}{|c|c|c|c|c|c|c|c|c|}
\hline \multirow[t]{2}{*}{ Characteristics } & \multicolumn{4}{|l|}{ Practices } & \multicolumn{4}{|l|}{ Attitudes } \\
\hline & Poor & Good & Chi2 & p-value & Poor & Good & Chi2 & $\begin{array}{l}\mathrm{p}- \\
\text { value }\end{array}$ \\
\hline$>1000$ & $22(9.95)$ & $\begin{array}{l}199 \\
(90.05)\end{array}$ & & & $4(1.81)$ & $\begin{array}{l}217 \\
(98.19)\end{array}$ & & \\
\hline $500-1000$ & $9(12.33)$ & 64 (87.67) & & & $6(8.22)$ & $67(91.78)$ & & \\
\hline$<500$ & $\begin{array}{l}12 \\
(16.00)\end{array}$ & $63(84.00)$ & & & $6(8.00)$ & 69 (92.00) & & \\
\hline Ethnicity & & & 5.34 & 0.151 & & & 6.76 & 0.124 \\
\hline Akan & $\begin{array}{l}28 \\
(13.02)\end{array}$ & $\begin{array}{l}187 \\
(86.98)\end{array}$ & & & $9(4.19)$ & $\begin{array}{l}206 \\
(95.81)\end{array}$ & & \\
\hline Ewe & $\begin{array}{l}10 \\
(16.13)\end{array}$ & $52(83.87)$ & & & $6(9.68)$ & $56(90.32)$ & & \\
\hline $\mathrm{Ga}$ & $1(3.03)$ & 32 (96.97) & & & $0(0.00)$ & $\begin{array}{l}33 \\
(100.00)\end{array}$ & & \\
\hline Others & $4(6.78)$ & 55 (93.22) & & & $1(1.69)$ & $58(98.31)$ & & \\
\hline Residence & & & 7.43 & 0.356 & & & 5.83 & 0.447 \\
\hline Ashanti & $8(15.38)$ & 44 (84.62) & & & $3(5.77)$ & 49 (94.23) & & \\
\hline Bono & $4(17.39)$ & 19 (82.61) & & & $2(8.70)$ & 21 (91.30) & & \\
\hline Central & $2(7.14)$ & $26(92.86)$ & & & $\begin{array}{l}3 \\
(10.71)\end{array}$ & 25 (89.29) & & \\
\hline Eastern & $6(13.33)$ & 39 (86.67) & & & $1(2.22)$ & 44 (97.78) & & \\
\hline Greater Accra & $9(7.32)$ & $\begin{array}{l}114 \\
(92.68)\end{array}$ & & & $4(3.25)$ & $\begin{array}{l}119 \\
(96.75)\end{array}$ & & \\
\hline Volta & $8(20.00)$ & $32(80.00)$ & & & $1(2.50)$ & 39 (97.50) & & \\
\hline Western & $1(6.67)$ & 14 (93.33) & & & $1(6.67)$ & 14 (93.33) & & \\
\hline Others & $5(11.63)$ & 38 (88.37) & & & $1(2.33)$ & 42 (97.67) & & \\
\hline
\end{tabular}

\subsection{Differences between Knowledge, Attitudes and Practices towards COVID-19 by Socio-demographics characteristics}

There was significant difference in knowledge on COVID-19 in relation to the occupation $(t=-2.984, p$-value $=$ $0.0030)$ of study participants. Again, significant differences existed in attitudes with respect to income level ( $F$ $=4.12, \mathrm{p}$-value $=0.0169)$ and ethnicity $(F=4.67, p$-value $=0.0032)$. Also, there was significant differences in 
practices towards the COVID-19 pandemic regarding gender $(t=4.712$, $p$-value $=0.0001)$, occupation $(t=$ $-3.320, \mathrm{p}$-value $=0.0010)$ and income level $(F=3.25$, $p$-value $=0.0400)$. (Table 6) 
Table 6

Differences between Knowledge, Attitudes and Practices towards COVID-19 by Socio-demographics characteristics

\begin{tabular}{|c|c|c|c|c|c|c|c|c|c|}
\hline \multirow[t]{2}{*}{ Characteristics } & \multicolumn{3}{|c|}{ Knowledge } & \multicolumn{3}{|c|}{ Attitudes } & \multicolumn{3}{|c|}{ Practices } \\
\hline & $\begin{array}{l}\text { Mean } \\
\pm \text { SD }\end{array}$ & $t / F$ & p-value & $\begin{array}{l}\text { Mean } \\
\pm \text { SD }\end{array}$ & $t / F$ & p-value & $\begin{array}{l}\text { Mean } \\
\pm \text { SD }\end{array}$ & $t / F$ & p-value \\
\hline Gender & & -1.016 & 0.3105 & & -0.569 & 0.5701 & & 4.712 & $\hat{0} .0001^{*}$ \\
\hline Female & $\begin{array}{l}18.10 \\
\pm 0.2\end{array}$ & & & $\begin{array}{l}5.25 \\
\pm 0.1\end{array}$ & & & $\begin{array}{l}9.71 \\
\pm 0.2\end{array}$ & & \\
\hline Male & $\begin{array}{l}18.36 \\
\pm 0.2\end{array}$ & & & $\begin{array}{l}5.31 \\
\pm 0.1\end{array}$ & & & $\begin{array}{l}8.63 \\
\pm 0.1\end{array}$ & & \\
\hline Age & & 1.36 & 0.2559 & & 2.58 & 0.0534 & & 1.86 & 0.1359 \\
\hline $18-25$ & $\begin{array}{l}17.91 \\
\pm 2.2\end{array}$ & & & $\begin{array}{l}5.08 \\
\pm 1.0\end{array}$ & & & $\begin{array}{l}8.76 \\
\pm 2.1\end{array}$ & & \\
\hline $26-33$ & $\begin{array}{l}18.30 \\
\pm 2.3\end{array}$ & & & $\begin{array}{l}5.33 \\
\pm 0.9\end{array}$ & & & $\begin{array}{l}8.92 \\
\pm 2.3\end{array}$ & & \\
\hline $34-41$ & $\begin{array}{l}18.37 \\
\pm 2.7\end{array}$ & & & $\begin{array}{l}5.41 \\
\pm 0.7\end{array}$ & & & $\begin{array}{l}9.34 \\
\pm 2.1\end{array}$ & & \\
\hline $42-64$ & $\begin{array}{l}18.88 \\
\pm 2.0\end{array}$ & & & $\begin{array}{l}5.31 \\
\pm 0.6\end{array}$ & & & $\begin{array}{l}9.62 \\
\pm 2.4\end{array}$ & & \\
\hline Occupation & & -2.984 & $0.0030^{*}$ & & -1.131 & 0.2587 & & -3.320 & $0.0010 *$ \\
\hline $\begin{array}{l}\text { Non-health } \\
\text { worker }\end{array}$ & $\begin{array}{l}17.94 \\
\pm 0.2\end{array}$ & & & $\begin{array}{l}5.24 \\
\pm 0.1\end{array}$ & & & $\begin{array}{l}8.71 \\
\pm 0.2\end{array}$ & & \\
\hline Health worker & $\begin{array}{l}18.67 \\
\pm 0.1\end{array}$ & & & $\begin{array}{l}5.35 \\
\pm 0.1\end{array}$ & & & $\begin{array}{l}9.46 \\
\pm 0.2\end{array}$ & & \\
\hline $\begin{array}{l}\text { Educational } \\
\text { level }\end{array}$ & & -1.007 & 0.3145 & & 0.085 & 0.9321 & & -0.704 & 0.4818 \\
\hline Secondary & $\begin{array}{l}17.62 \\
\pm 1.0\end{array}$ & & & $\begin{array}{l}5.31 \\
\pm 0.2\end{array}$ & & & $\begin{array}{l}8.62 \\
\pm 0.5\end{array}$ & & \\
\hline Tertiary & $\begin{array}{l}18.29 \\
\pm 0.1\end{array}$ & & & $\begin{array}{l}5.29 \\
\pm 0.1\end{array}$ & & & $\begin{array}{l}9.05 \\
\pm 0.1\end{array}$ & & \\
\hline Religion & & 1.83 & 0.1623 & & 0.24 & 0.7850 & & 0.49 & 0.6136 \\
\hline Christianity & $\begin{array}{l}18.30 \\
\pm 2.4\end{array}$ & & & $\begin{array}{l}5.29 \\
\pm 0.9\end{array}$ & & & $\begin{array}{l}9.04 \\
\pm 2.2\end{array}$ & & \\
\hline Islamic & $\begin{array}{l}17.60 \\
\pm 1.8\end{array}$ & & & $\begin{array}{l}5.40 \\
\pm 0.5\end{array}$ & & & $\begin{array}{l}8.60 \\
\pm 1.8\end{array}$ & & \\
\hline
\end{tabular}

Others* refers to Ahafo, Bono East, North East, Northern, Oti, Savannah, Upper East, Upper West and Western North regions. 


\begin{tabular}{|c|c|c|c|c|c|c|c|c|c|}
\hline \multirow[t]{2}{*}{ Characteristics } & \multicolumn{3}{|c|}{ Knowledge } & \multicolumn{3}{|c|}{ Attitudes } & \multicolumn{3}{|c|}{ Practices } \\
\hline & $\begin{array}{l}\text { Mean } \\
\pm \text { SD }\end{array}$ & $t / F$ & p-value & $\begin{array}{l}\text { Mean } \\
\pm \text { SD }\end{array}$ & $t / F$ & p-value & $\begin{array}{l}\text { Mean } \\
\pm \text { SD }\end{array}$ & $t / F$ & p-value \\
\hline Others & $\begin{array}{l}16.00 \\
\pm 1.7\end{array}$ & & & $\begin{array}{l}5.00 \\
\pm 1.0\end{array}$ & & & $\begin{array}{l}10.00 \\
\pm 1.7\end{array}$ & & \\
\hline $\begin{array}{l}\text { Income level } \\
\text { (C) }\end{array}$ & & 2.30 & 0.1018 & & 4.12 & $0.0169 *$ & & 3.25 & $0.0400 *$ \\
\hline$>1000$ & $\begin{array}{l}18.47 \\
\pm 2.4\end{array}$ & & & $\begin{array}{l}5.39 \\
\pm 0.8\end{array}$ & & & $\begin{array}{l}9.16 \\
\pm 2.2\end{array}$ & & \\
\hline $500-1000$ & $\begin{array}{l}18.10 \\
\pm 2.2\end{array}$ & & & $\begin{array}{l}5.14 \\
\pm 1.0\end{array}$ & & & $\begin{array}{l}9.23 \\
\pm 2.1\end{array}$ & & \\
\hline$<500$ & $\begin{array}{l}17.83 \\
\pm 2.5\end{array}$ & & & $\begin{array}{l}5.12 \\
\pm 1.0\end{array}$ & & & $\begin{array}{l}8.47 \\
\pm 2.1\end{array}$ & & \\
\hline Ethnicity & & 0.24 & 0.8660 & & 4.67 & $0.0032^{*}$ & & 2.58 & 0.0536 \\
\hline Akan & $\begin{array}{l}18.24 \\
\pm 2.5\end{array}$ & & & $\begin{array}{l}5.38 \\
\pm 0.9\end{array}$ & & & $\begin{array}{l}8.90 \\
\pm 2.2\end{array}$ & & \\
\hline Ewe & $\begin{array}{l}18.45 \\
\pm 2.0\end{array}$ & & & $\begin{array}{l}4.92 \\
\pm 1.1\end{array}$ & & & $\begin{array}{l}9.19 \\
\pm 2.4\end{array}$ & & \\
\hline $\mathrm{Ga}$ & $\begin{array}{l}18.03 \\
\pm 2.5\end{array}$ & & & $\begin{array}{l}5.36 \\
\pm 0.7\end{array}$ & & & $\begin{array}{l}9.97 \\
\pm 2.0\end{array}$ & & \\
\hline Others & $\begin{array}{l}18.27 \\
\pm 2.2\end{array}$ & & & $\begin{array}{l}5.29 \\
\pm 0.6\end{array}$ & & & $\begin{array}{l}8.85 \\
\pm 1.9\end{array}$ & & \\
\hline $\begin{array}{l}\text { Region of } \\
\text { residence }\end{array}$ & & 0.67 & 0.6992 & & 0.31 & 0.9492 & & 0.77 & 0.6141 \\
\hline Ashanti & $\begin{array}{l}18.25 \\
\pm 2.1\end{array}$ & & & $\begin{array}{l}5.25 \\
\pm 0.9\end{array}$ & & & $\begin{array}{l}8.79 \\
\pm 2.2\end{array}$ & & \\
\hline Bono & $\begin{array}{l}18.56 \\
\pm 1.8\end{array}$ & & & $\begin{array}{l}5.26 \\
\pm 1.1\end{array}$ & & & $\begin{array}{l}9.35 \\
\pm 3.1\end{array}$ & & \\
\hline Central & $\begin{array}{l}18.86 \\
\pm 1.5\end{array}$ & & & $\begin{array}{l}5.14 \\
\pm 1.3\end{array}$ & & & $\begin{array}{l}9.29 \\
\pm 2.2\end{array}$ & & \\
\hline Eastern & $\begin{array}{l}18.16 \\
\pm 2.2\end{array}$ & & & $\begin{array}{l}5.27 \\
\pm 0.8\end{array}$ & & & $\begin{array}{l}8.87 \\
\pm 2.1\end{array}$ & & \\
\hline Greater Accra & $\begin{array}{l}18.07 \\
\pm 2.5\end{array}$ & & & $\begin{array}{l}5.28 \\
\pm 0.8\end{array}$ & & & $\begin{array}{l}9.28 \\
\pm 2.0\end{array}$ & & \\
\hline Volta & $\begin{array}{l}18.55 \\
\pm 2.2\end{array}$ & & & $\begin{array}{l}5.33 \\
\pm 0.8\end{array}$ & & & $\begin{array}{l}8.80 \\
\pm 2.5\end{array}$ & & \\
\hline Western & $\begin{array}{l}18.67 \\
\pm 2.6\end{array}$ & & & $\begin{array}{l}5.40 \\
\pm 0.8\end{array}$ & & & $\begin{array}{l}9.20 \\
\pm 2.1\end{array}$ & & \\
\hline
\end{tabular}

Others* refers to Ahafo, Bono East, North East, Northern, Oti, Savannah, Upper East, Upper West and Western North regions. 


\begin{tabular}{|c|c|c|c|c|c|c|c|c|c|}
\hline \multirow[t]{2}{*}{ Characteristics } & \multicolumn{3}{|c|}{ Knowledge } & \multicolumn{3}{|c|}{ Attitudes } & \multicolumn{3}{|c|}{ Practices } \\
\hline & $\begin{array}{l}\text { Mean } \\
\pm \text { SD }\end{array}$ & $t / F$ & p-value & $\begin{array}{l}\text { Mean } \\
\pm \text { SD }\end{array}$ & $t / F$ & p-value & $\begin{array}{l}\text { Mean } \\
\pm \text { SD }\end{array}$ & $t / F$ & p-value \\
\hline Others & $\begin{array}{l}17.98 \\
\pm 3.0\end{array}$ & & & $\begin{array}{l}5.42 \\
\pm 0.7\end{array}$ & & & $\begin{array}{l}8.63 \\
\pm 1.9\end{array}$ & & \\
\hline
\end{tabular}

\subsection{Multiple linear regression on factors associated with COVID-19 practices}

A multiple linear regression analysis depicted that male gender (vs female, $\beta=-1.276$, $p$-value $=<0.00001$ ), income level ( $($ ) of less than 500 (vs above $1000, \beta=-0.845$, $p$-value $=0.017$ ) were significantly associated with lower practice score. (Table 7). However, 42-64 age category (vs 18-25-year group, $\beta=1.128, p$-value $=$ 0.028 ), health worker as an occupation (vs non-health worker, $\beta=0.816, p$-value $=0.001$ ) and belonging to an Ga ethnic group (vs Akan ethnic group, $\beta=0.89$, $p$-value $=0.027$ ) were significantly associated with higher practice score. (Table 7)

Table 7

Results of multiple linear regression on factors associated with COVID-19 practices

\begin{tabular}{|lllll|}
\hline Variable & Coefficient & Standard error & t & p-value \\
\hline Gender (Male vs. Female) & -1.276 & 0.241 & -5.29 & $<0.0001$ \\
\hline Age (42-64 vs. 18-25) & 1.128 & 0.511 & 2.21 & 0.028 \\
\hline Occupation (Health worker vs. Non-health worker) & 0.816 & 0.235 & 3.48 & 0.001 \\
\hline Income in GHç (Less than 500 vs. Above 1000) & -0.845 & 0.353 & -2.39 & 0.017 \\
\hline Ethnicity (Ga vs. Akan) & 0.891 & 0.401 & 2.22 & 0.027 \\
\hline
\end{tabular}

\section{Discussion}

This study investigated the knowledge, attitudes and practices of Ghanaians towards the novel coronavirus disease through an online survey. Averagely, participants of this study showed a high knowledge of the disease. This finding was not different from a similar study conducted among the Egyptian population (12). Additionally, an extremely higher mean score of knowledge was recorded in a study conducted among Chinese residents (15). All these results show the concern that was attached to the disease. Besides, the high COVID-19 knowledge level may also have resulted from the educational level of participants took part in the study (16).

Again, our study revealed that health workers had significantly higher knowledge score compared to nonhealth workers. This result is expected since health workers are the front-liners in the fight against the coronavirus pandemic. However, a study conducted by Yue et al. showed that being a health care worker had a 
significant impact on COVID-19 knowledge, but there was no significant difference in knowledge of health workers and non-health care workers (17). Significant differences in COVID-19 knowledge rather existed in age groups, marital status, gender and educational level (17).

The findings of our study depicted a high mean percentage attitude score of almost $90 \%$. Similar result was reported in a population based study done in Iran (18). Further, both recent and previous studies conducted within Africa and other parts of the world within this pandemic period have reported high positive attitudes towards the novel coronavirus disease $(15-17,19)$. Knowledge is usually associated with attitudes. Therefore, the high COVID-19 knowledge among participants from different countries may have influenced the general attitude of people worldwide. A study conducted in Nigeria confirms a significant relationship between COVID19 knowledge level and attitudes towards COVID-19 preventive practices (16).

Earlier modelling studies had predicted the severity of the novel coronavirus pandemic globally; the severity is expected to be intense in low-income settings and among people earning low income $(20,21)$. In our study, we found that attitudes towards COVID-19 was significantly associated with income level of participants. And, it was confirmed in our study that positive attitude towards the COVID-19 was significantly higher among participants who received higher income compared to those who received lower income. Also, positive attitudes towards COVID-19 were found among majority of above $\$ 1000$ income earners. Therefore, it is obvious that negative attitudes among low-income earners may be the reason why the severity of the COVID19 preventive items, such as nose masks and sanitizers.

In a study (22) conducted among ethnic minorities in Hong Kong, participants expressed some misconceptions on the prevention of novel coronavirus disease; however, they had positive attitudes towards the approved preventive measures of the infection including wearing of masks and social distancing. Though in our study, ethnicity was not associated with attitudes towards COVID-19, significant differences in attitude was among ethnic groups, implying that an ethnic group had better attitudes towards COVID-19 compared to the others. These findings are expected because earlier studies (23) have reported a relationship between ethnicity and spread of COVID-19 disease. This observation suggests that the cultural and socio-economic characteristics of an ethnic group significantly affects their attitudes toward a disease; whether positive or negative.

The mean percentage of COVID-19 preventive practices in our study was found to be $69.2 \%$. This outcome was similar to a recent research in Nigeria that depicted an average percentage of $68.0 \%$ (16). Nonetheless, a study published after some few months of the COVID-19 pandemic revealed that over $95.0 \%$ of study participants engaged in all COVID-19 preventive practices (15). The discrepancies in the average practices may be due to the period, and countries where the studies were conducted. The earlier study (16) and our study were conducted in West African countries during the national lockdown, which was many months into the COVID-19 pandemic; however, the latter was conducted in the early period of the epidemic in China.

Also, in this study, practices towards the novel coronavirus was significantly associated with gender. Besides, although our study found that majority of males were found to be involved in good practices of COVID-19, female participants significantly adhere to COVID-19 preventive measures compared to their male counterparts. The findings of this study synchronizes with another, which revealed that men were associated 
with lower practice of COVID-19 preventive measures, including wearing nose masks and going to crowded places (15). A study by Erfani et al. also confirms the high practice of COVID-19 preventive measures among females compared to males (18). Additionally, a research (24) conducted to study gender differences in COVID-19 linked beliefs and behaviour revealed that, generally, females perceived the pandemic as a serious health issue and subsequently comply with all the preventive measures compared to males. This accounts for the considerable difference in COVID-19 mortality cases between women and men.

This study found occupation and income level of study participants to be significantly associated with COVID19 preventive practices. As expected, health workers practiced COVID-19 preventive measures compared to non-health workers. Erfani et al. study (18) on COVID-19 found results similar to our study, where being a health care provider was associated with adequate knowledge, attitude and preventive practices towards COVID-19 infection. The population working in health care related facilities are the forefront of the coronavirus virus pandemic; therefore, it is highly expected of these workers to practice COVID-19 preventive measures. According to a recent Chinese study, COVID-19 preventive measures were practiced by participants who earned higher income (22). However, the results of this study revealed that participants who earned between $\$ 500-$ 1000 as income practiced COVID-19 preventive measures compared to those received less than 500 and above $\$ 1000$. Furthermore, our study showed a strong association between income level of above $\$ 1000$ and lower practice score. These results suggest the unpredictability of human behaviour, which may be affected by factors such as knowledge and complacency.

This study, finally, found out that being within the age category of 42-64 years and belonging to a Ga ethnic group are associated with higher COVID-19 preventive practice score. The numerous data that churned out at the early stage of the COVID-19 pandemic revealed that the older age group were at a high risk compared to the youth (25). So, educational and preventive campaigns were all targeted at this age bracket. This may be the reason for the higher practice score among them (26). The Ga ethnic group are inhabitants of Accra, the epicenter of the novel coronavirus in Ghana. In essence, it was expected that this group may adhere more to the preventive measures compared to the other ethnic groups in Ghana.

\section{Limitation of the study}

Due to the COVID-19 pandemic, the study was limited to only participants who could read and write, since the survey was made available on solely social media platforms.

\section{Conclusions}

In summary, our research suggests that knowledge and attitudes towards novel coronavirus disease was high among the Ghanaian population; however, preventive practices towards the infection was just above average. Occupation and income level had a significant relation with knowledge and attitudes towards COVID-19 infection, respectively. Also, COVID-19 preventive practices had significant association with gender, income level, occupation, age and ethnic group. Educational interventions on COVID-19 should be targeted towards the population involved in non-health related occupation. Good attitudes towards the pandemic should be encouraged among the low-income earners of the population. The male gender and low-income earners should be admonished to practice measures towards the curbing of the novel coronavirus disease. 


\section{Abbreviations}

COVID-19

Coronavirus disease 2019

GoG

Government of Ghana

ANOVA

Analysis of variance

KAP

Knowledge, Attitude and Practices

\section{Declarations}

\section{Ethics approval and consent to participate}

The Ghana Health Service Ethical Review Committee (GHS-ERC) guidelines and standard operating procedures exempt studies that present minimal risks such as online surveys, public behaviour observations and interviews from ethical review and clearance. Hence, the study did not require ethical approval. However, study participants' anonymity and confidentiality was ensured since the survey did not require any identity before partaking. An informed consent section was created as part of the questionnaire to seek consent before respondents proceeded to complete the online study questionnaire.

\section{Consent for publication}

Not applicable

\section{Availability of data and material}

The study data and materials will be provided upon request.

\section{Competing of Interest}

The authors declare no competing interest regarding the publication of this study

\section{Funding}

The authors received no funds for the study

\section{Authors' Contributions}

PAT designed the study and searched literature. KAW, PAT and KO wrote the protocol and methods. PAT, KAW, $\mathrm{KO}, \mathrm{BNT}, \mathrm{AAY}, \mathrm{PEK}$ designed the data collection tool and collected data. PAT, KAW and KO managed the preliminary statistical analysis and wrote the first draft of the manuscript. PAT, KAW and KO managed the final statistical analysis of the study and wrote the final manuscript. All authors read and gave approval for the final manuscript. 


\section{Acknowledgements}

The authors express their utmost gratitude to all study participants who took time out of their busy schedules to complete the questionnaire for the survey hence making the study successful.

\section{References}

1. Wang C, Horby PW, Hayden FG, Gao GF. A novel coronavirus outbreak of global health concern [Review of A novel coronavirus outbreak of global health concern]. The Lancet. 2020;395(10223):470-3.

2. World Health Organization. COVID-19 Public Health Emergency of International Concern (PHEIC) Global research and innovation forum [Internet]. 2020 [cited 2020 Feb 12]. Available from: https://www.who.int/who-documents-detail/covid-19-public-health-emergency-of-international-concern(ph.

3. World Health Organization. WHO announces COVID-19 outbreak a pandemic [Internet]. 2020. Available from: http://www.euro.who.int/en/health-topics/health-emergencies/coronavirus-covid19/news/news/2020/3/who-announces-covid-19-outbreak-a-pandemic.

4. Citi News Room. Two cases of coronavirus confirmed in Ghana. Citinewsroom - Comprehensive News in Ghana, Current Affairs, Business News, Headlines, Ghana Sports, Entertainment, Politics, Articles, Opinions, Viral Content. [Internet]. 2020 [cited 2020 Mar 12]. Available from: https://citinewsroom.com/2020/03/two-cases-of-coronavirus-confirmed-in-ghana/.

5. Citi News Room. Coronavirus: Government bans religious activities, funerals, all other public gatherings. Citinewsroom - Comprehensive News in Ghana, Current Affairs, Business News, Headlines, Ghana Sports, Entertainment, Politics, Articles, Opinions, [Internet]. 2020 [cited 2020 Mar 15]. Available from: https://citinewsroom.com/2020/03/government-bans-church-activities-funerals-all-other-publicgatherings/.

6. Ghana Health Service. COVID-19 Updates | Ghana. [Internet]. 2020. Available from: https://www.ghanahealthservice.org/covid19/.

7. Tachfouti N, Slama K, Berraho M, Nejjari C. The impact of knowledge and attitudes on adherence to tuberculosis treatment: a case-control study in a Moroccan region. The Pan African Medical Journal. 2012;12(52).

8. Ajilore K, Atakiti I, Onyenankeya K. College students' knowledge, attitudes and adherence to public service announcements on Ebola in Nigeria: Suggestions for improving future Ebola prevention education programmes. Health Education Journal. 2017;76(6):648-60.

9. Person B, Sy F, Holton K, Govert B, Liang A. Fear and stigma: the epidemic within the SARS outbreak. Emerg Infect Dis. 2004;10(2):358-63.

10. Tao N, Sussman S, Nieto J, Tsukamoto H, Yuan J-M. Demographic characteristics of hospitalized patients with alcoholic liver disease and pancreatitis in los angeles county. Alcohol Clin Exp Res. 2003;27(11):1798-804.

11. Zhong B-L, Luo W, Li H-M, Zhang Q-Q, Liu X-G, Li W-T, et al. Knowledge, attitudes, and practices towards COVID-19 among Chinese residents during the rapid rise period of the COVID-19 outbreak: a quick online cross-sectional survey. International Journal of Biological Sciences. 2020;16(10):1745-52. 
12. Abdelhafiz AS, Mohammed Z, Ibrahim ME, Ziady HH, Alorabi M, Ayyad M, et al. Knowledge, Perceptions, and Attitude of Egyptians Towards the Novel Coronavirus Disease (COVID-19). Journal of Community Health. 2020.

13. Erfani A, Shahriarirad R, Ranjbar K, Mirahmadizadeh A, Moghadami M. Knowledge, Attitude and Practice toward the Novel Coronavirus (COVID-19) Outbreak: A Population-Based Survey in Iran. WHO. 2020.

14. Abuya T, Austrian K, Isaac A, Kangwana B, Mbushi F, Muluve E, et al. COVID-19-related knowledge, attitudes, and practices in urban slums in Nairobi, Kenya: Study description. 2020.

15. Zhong B-L, Luo W, Li H-M, Zhang Q-Q, Liu X-G, Li W-T, et al. Knowledge, attitudes, and practices towards COVID-19 among Chinese residents during the rapid rise period of the COVID-19 outbreak: a quick online cross-sectional survey. Int J Biol Sci. 2020;16(10):1745-52.

16. Reuben RC, Danladi MMA, Saleh DA, Ejembi PE. Knowledge, Attitudes and Practices Towards COVID-19: An Epidemiological Survey in North-Central Nigeria. J Community Health. 2021 Jun;46(3):457-70.

17. Yue S, Zhang J, Cao M, Chen B. Knowledge. Attitudes and Practices of COVID-19 Among Urban and Rural Residents in China: A Cross-sectional Study. J Community Health. 2021 Apr;46(2):286-91.

18. Erfani A, Shahriarirad R, Ranjbar K, Mirahmadizadeh A, Moghadami M. Knowledge. Attitude and Practice toward the Novel Coronavirus (COVID-19) Outbreak: A Population-Based Survey in Iran [Internet]. nCoV; 2020 Mar [cited 2021 Jun 11]. Available from: http://www.who.int/bulletin/online_first/20-256651.pdf.

19. Abbasi-Kangevari M, Kolahi A-A, Ghamari S-H, Hassanian-Moghaddam H. Public Knowledge, Attitudes, and Practices Related to COVID-19 in Iran: Questionnaire Study. JMIR Public Health Surveill. 2021 Feb 23;7(2):e21415.

20. Walker P, Whittaker C, Watson O, Baguelin M, Ainslie K, Bhatia S, et al. Report 12: The global impact of COVID-19 and strategies for mitigation and suppression [Internet]. Imperial College London; 2020 Mar [cited 2021 Jun 12]. Available from: http://spiral.imperial.ac.uk/handle/10044/1/77735.

21. Truelove S, Abrahim O, Altare C, Lauer SA, Grantz KH, Azman AS, et al. The potential impact of COVID-19 in refugee camps in Bangladesh and beyond: A modeling study. Parmar P, editor. PLoS Med. 2020 Jun;16(6):e1003144. 17(.

22. Wong CL, Chen J, Chow KM, Law BMH, Chan DNS, So WKW, et al. Knowledge, Attitudes and Practices Towards COVID-19 Amongst Ethnic Minorities in Hong Kong. IJERPH. 2020 Oct 27;17(21):7878.

23. Pareek M, Bangash MN, Pareek N, Pan D, Sze S, Minhas JS, et al. Ethnicity and COVID-19: an urgent public health research priority. The Lancet. 2020 May;395(10234):1421-2.

24. Galasso V, Pons V, Profeta P, Becher M, Brouard S, Foucault M. Gender differences in COVID-19 attitudes and behavior: Panel evidence from eight countries. Proc Natl Acad Sci USA. 2020 Nov;3(44):27285-91. 117 .

25. Undurraga EA, Chowell G, Mizumoto K. COVID-19 case fatality risk by age and gender in a high testing setting in Latin America: Chile, March-August 2020. Infect Dis Poverty. 2021 Dec;10(1):11.

26. Kim JK, Crimmins EM. How does age affect personal and social reactions to COVID-19: Results from the national Understanding America Study. Sykes BL, editor. PLoS ONE. 2020 Nov 10;15(11):e0241950.

\section{Figures}




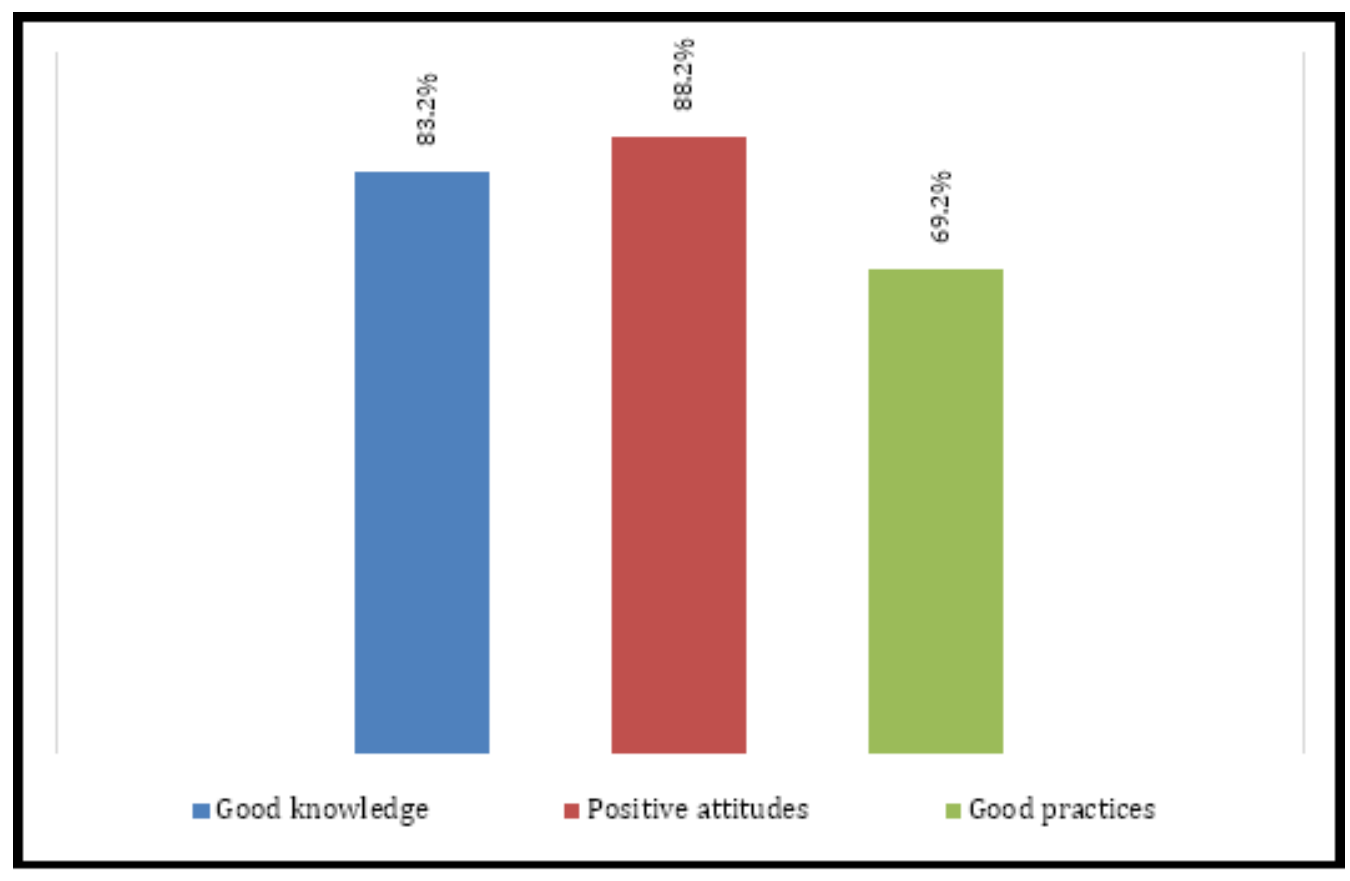

\section{Figure 1}

Percentage of adequate knowledge, positive attitude and good practices among participants

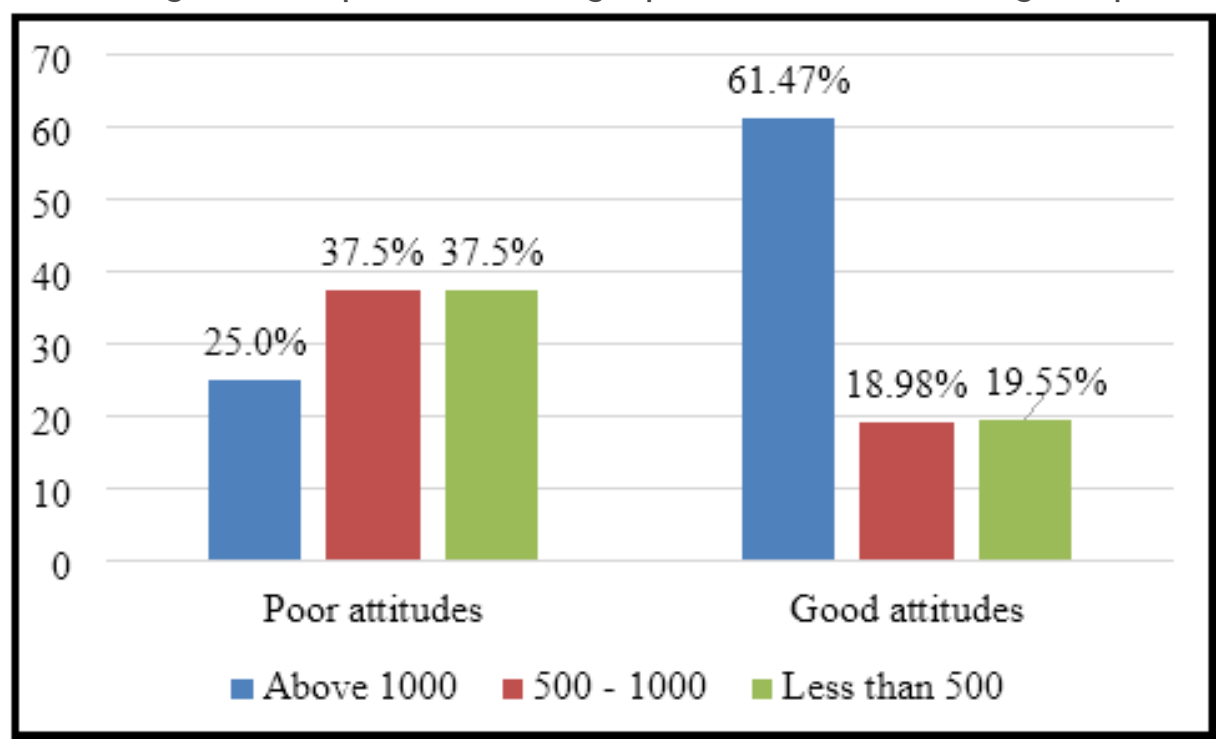

\section{Figure 2}

Level of participants' attitudes towards COVID-19 by income level 


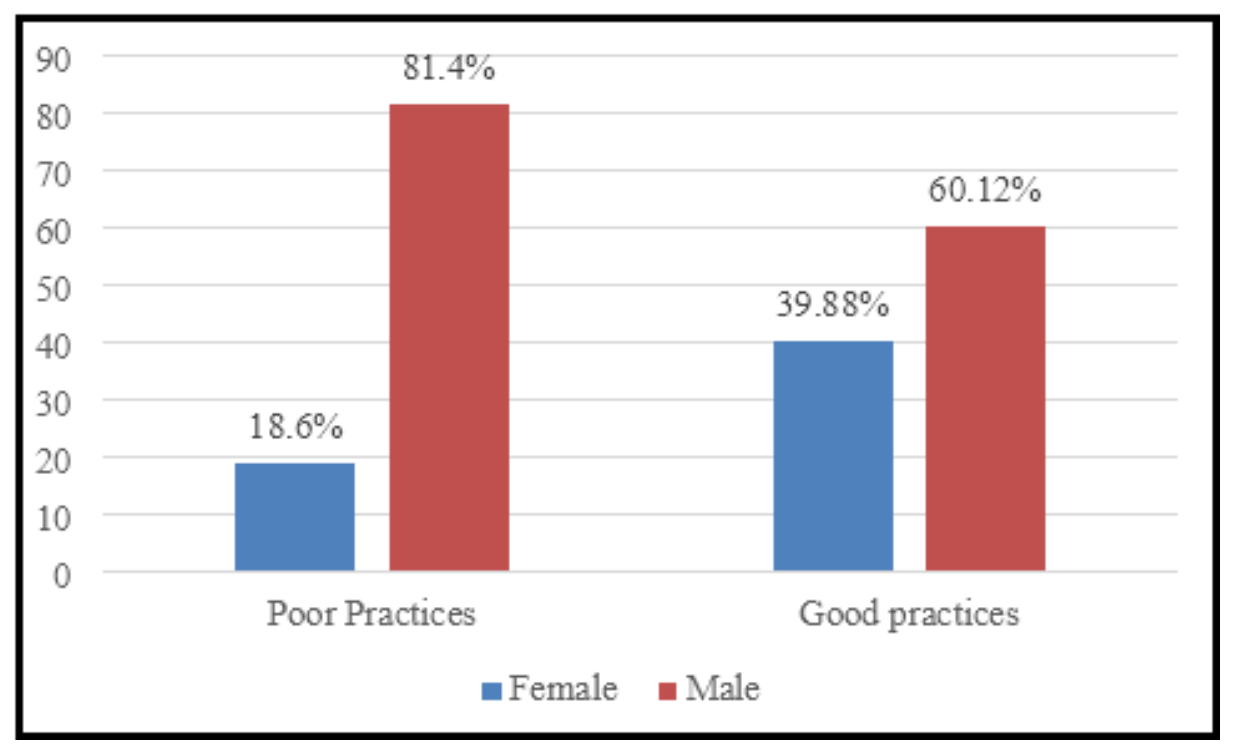

\section{Figure 3}

Level COVID-19 preventive practices among participants by gender

\section{Supplementary Files}

This is a list of supplementary files associated with this preprint. Click to download.

- StudyQuestionnaire.pdf 